\title{
Evaluation of platelet indices as a useful marker in papillary thyroid carcinoma
}

\author{
Dincel $\mathrm{O}^{1}$, Bayraktar $\mathrm{C}^{2}$ \\ Adiyaman University Research and Education Hospital, Department of General Surgery, \\ Adiyaman, Turkey.cem_dr23@hotmail.com
}

\section{ABSTRACT}

OBJECTIVES: Our aim was to investigate platelets (PLT) and their indices - mean platelet volume (MPV), platelet distribution width (PDW) and plateletcrit (PCT) - in the differential diagnosis of benign and malignant thyroid disorders. BACKGROUND: Platelet and platelet indices recently started to be defined as an useful clinical marker in cancer patients. Their higher reproducibility rates and low cost provide them a wide range of usage.

MATERIAL AND METHODS: One-hundred and sixty patients were divided into three groups retrospectively. Group 1: papillary thyroid carcinoma patients $(n=65)$. Group 2: multi-nodular goiter patients $(n=65)$. Group 3: normal healthy subjects $(n=30)$. PLT, MPV, PDW and PCT measurements were compared between groups, and $p<0.05$ was accepted as significant.

RESULTS: PDW was found to be significantly decreased in Group $1(p<0.01)$, and PCT was observed to be significantly increased in Group $1(p<0.01)$. There were no significant differences in PLT and MPV results. CONCLUSION: Statistically significant increases in PCT and decreases in PDW were observed in papillary thyroid cancer patients when compared with other groups. Platelet indices may be used as useful clinical markers in the differential diagnosis of benign and malignant thyroid disorders (Tab. 1, Fig. 2, Ref. 21). Text in PDF www.elis.sk. KEY WORDS: papillary thyroid cancer, mean platelet volume, platelet distribution width, plateletcrit.

\section{Introduction}

The prevalence of thyroid nodules ranges from 3-7 \% by palpation and $19-67 \%$ by high resolution ultrasonography (1). Nearly half of the nodules are benign, and they are found in women at a rate of four times higher than men. The rate of newly formed nodules is $0.1 \%$ per year from early life (2). A multi-nodular goiter (MNG) can be defined as an enlargement of a thyroid gland that has multiple nodules and it is known as a benign disease with a low malignancy risk since the past (3).

Thyroid cancers are the most common endocrine malignancy, and papillary thyroid carcinoma (PTC) is the most common form among them. The annual incidence of disease was stated in women and men to be 2.3 and 0.9 cases per 100,000, respectively (4). PTC is two and half times more frequent in women than men. Patients between the age of 20 and 45 have a good long-term prognosis, and mortality rate for the cancer is low in this age range (5).

Proinflammatory factors, chemokines, growth factors and platelets have an important role in the basis of inflammation, which can cause cancer to develop (6). New hematologic analyzers fa-

${ }^{1}$ Adiyaman University Research and Education Hospital, Department of General Surgery, Adiyaman, Turkey, and ${ }^{2}$ Adiyaman University Research and Education Hospital, Department of Otolaryngology Head and Neck Surgery, Adiyaman, Turkey

Address for correspondence: C. Bayraktar, MD, Adiyaman University Research and Education Hospital, Department of Otolaryngology Head and Neck Surgery, Adiyaman 02000, Turkey.

Phone: +90.532.2627781, Fax: +90.416.2145599 cilitate the evaluation of some parameters in addition to platelet (PLT) count. Platelet distribution width (PDW), plateletcrit (PCT) and mean platelet volume (MPV) are known as platelet indices.

Mean platelet volume shows the average platelet volume but does not reflect the microscopic changes. PDW is a coefficient of variation of the platelet volume average, and its high values indicate labile volumetric difference when compared with normal individuals. Some automatic hematologic analyzers use MPV and platelet values to calculate the PCT, which shows the platelet mass in a unit of volume like hematocrit for erythrocytes (7-9).

In recent years, it has been suggested that PLT and platelet indices could be used as an inflammatory marker in cancer patients in addition to use in cardiovascular, cerebrovascular, thromboembolic and inflammatory diseases. Low cost and high reproducibility rates enhance the usability of PLT and platelet indices (10).

In the present study, we aim to investigate the availability of PLT and platelet indices like MPV, PDW and PCT in the differential diagnosis of benign and malignant thyroid disorders.

\section{Material and methods}

This retrospective study was conducted in Adiyaman University Research and Education Hospital with patients who had undergone total thyroidectomy between May 2012 and March 2016. The Ethical Committee approval was obtained. Pathology results reporting papillary thyroid cancer and multinodular goiter were detected, and patient records indicating age, sex and preoperative hematologic values were recorded from patient charts. Patients 
Tab. 1. Demographic features and laboratory results of the study groups.

\begin{tabular}{|c|c|c|c|c|c|}
\hline & & $\begin{array}{c}\text { Group } 1 \\
\text { (PTC, } n=65)\end{array}$ & $\begin{array}{c}\text { Group } 2 \\
(\mathrm{MNG}, \mathrm{n}=65)\end{array}$ & $\begin{array}{c}\text { Group } 3 \\
(\text { Control, } n=30)\end{array}$ & $P$ value \\
\hline Age (years) & & $42.40 \pm 13.03$ & $45.29 \pm 12.22$ & $41.43 \pm 12.20$ & $0.27 \dagger$ \\
\hline \multirow{2}{*}{ Sex } & Male & $9(13 \%)$ & $10(15 \%)$ & $6(20 \%)$ & \multirow{2}{*}{$0.74^{¥}$} \\
\hline & Female & $56(86 \%)$ & $55(84 \%)$ & $24(80 \%)$ & \\
\hline PLT & & $243.75 \pm 56.53$ & $253.94 \pm 51.18$ & $248.79 \pm 61.12$ & $0.57 \dagger$ \\
\hline MPV & & $8.64 \pm 1.88$ & $8.20 \pm 1.73$ & $8.22 \pm 1.26$ & $0.36 \neq$ \\
\hline PDW & & $18.24 \pm 2.34$ & $19.34 \pm 2.24$ & $19.33 \pm 1.24$ & $<0.01 \ddagger^{* *}$ \\
\hline PCT & & $0.24 \pm 0.05 \mathbf{a}$ & $0.19 \pm 0.04 \mathbf{b}$ & $0.17 \pm 0.02 \mathbf{b}$ & $<0.01 \dagger^{*}$ \\
\hline
\end{tabular}

† One-way ANOVA test, ¥ Chi-square test, $\ddagger$ Kruskal Wallis test PLT - platelet, MPV - mean platelet volume, PDW - platelet distribution width, PCT - plateletcrit, * a, b letters show group differences (Post hoc test - Tukey HSD test), ** Paired comparisons for PDW performed with Mann-Whitney U test (Group 1 - Group 2 = p < 0.05; Group 1 - Group $3=p<0.05$; Group 2 - Group $3=p>0.05$ )

who had diabetes, hypertension, chronic inflammatory disease, autoimmune disease and impairment in hepatic, renal or hematologic laboratory findings were excluded from the study. The study patients were divided into 3 groups: Group 1 (PTC, $n=65$ ), Group 2 (MNG, $n=65$ ) and Group 3 (healthy controls, $n=30$ ). Platelets and their indices were compared in both groups. Blood samples were taken from the antecubital vein and collected into tubes containing ethylenediaminetetraacetic acid (EDTA). Measurements of platelets and their indices were performed with an automated hematologic analyzer. All MPV measurements were performed 120 minutes after sampling in all subjects for standardization as stated by Lance et al. for optimal measurements (11).

\section{Statistical analysis}

Data was analyzed using Statistical Package for Social Sciences version 15.0 (SPSS Inc, Chicago, IL, USA). The distribution analyses of age and complete blood count parameters of both groups were performed with the Kolmogorow-Smirnov test. Categorical variables were assessed with the Chi-square test. One-way ANOVA (analysis of variance) was used as a parametric test to evaluate differences between the three groups, and the post-hoc Tukey HSD test was performed for the significant comparisons. The Kruskal Wallis test was used if the distribution was not normal in groups. Intragroup paired comparisons were made with the Mann-Whitney $\mathrm{U}$ test to define the significance between groups. A p-value $<0.05$ was accepted as statistically significant.

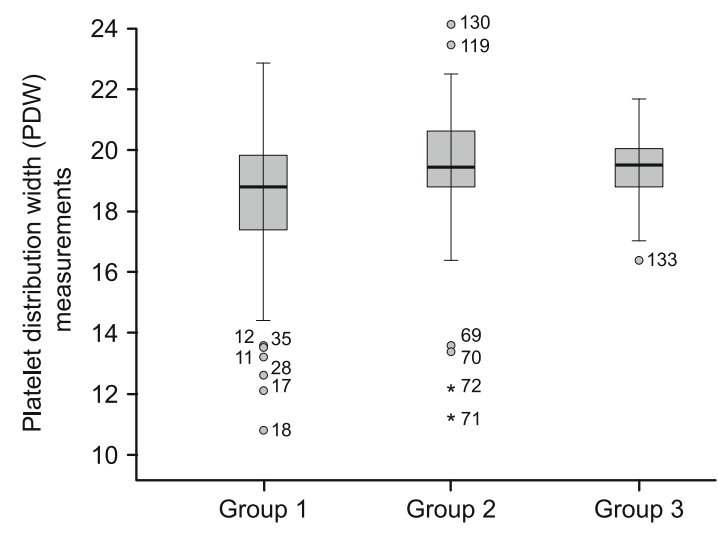

Fig. 1. The box-plots of platelet distrubution width (PDW) measurements in groups.

\section{Results}

There were no significant differences in age-sex, PLT or MPV values between groups ( $p>0.05)$. PDW was found to be significantly decreased in Group 1 ( $\mathrm{p}<0.01$ ). In paired comparisons for PDW, the significance was in Group1 - Group2 and Group1 - Group3 comparisons ( $<0.05)$. There was no significance in Group2 - Group3 comparison ( $\mathrm{p}>0.05$ ). PCT was observed to be significantly increased in Group 1 when compared with the other groups ( $\mathrm{p}<0.01$ ) (Tab. 1). The box-plots of PDW and PCT, which show the distribution of groups, were shown in Figures 1 and 2.

\section{Discussion}

The core findings of this study are i) MPV and PLT measurements were not significantly different from controls in PTC patients, ii) PDW values were found to be significantly decreased and iii) PCT measurements were observed to be significantly increased in PTC patients.

Platelet count was investigated in several different organ cancer studies. For instance, PLT levels were observed to be significantly lower in cases of non-small cell lung cancer and epithelial ovarian cancer, but no change was observed in breast and colon cancer (12-16).

The main mechanism of cancer pathophysiology is known to be genetic factors, but inflammatory response is also important. Local tumor-related inflammation can be seen in the preoperative

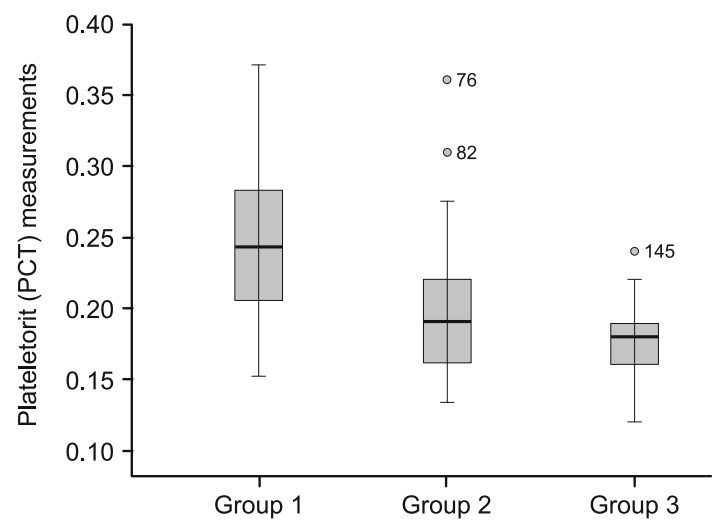

Fig. 2. The box-plots of plateletcrit (PCT) measurements in groups. 
period. Increased proinflammatory cytokines induce proliferation, and megakaryocytes are converted to platelets (17).

Platelets supply the production and release of vascular endothelial growth factor (VEGF), which plays a role in tumor angiogenesis and inflammation (18). In literature, high platelet levels were found in association with higher recurrence risk, later stages and metastasis in several types of cancers (15). To the best of our knowledge, this is the first study to investigate the PLT levels in PTC patients, and we could find no significant difference between PTC and MNG patients.

Larger platelets are more active than small ones, and MPV shows platelet function. MPV has been investigated in many types of cancer. Recently, it has been shown that MPV levels were significantly increased in endometrial cancer, ovarian cancer, colorectal cancer and gastric cancer when compared with healthy controls. Baldane et al reported significantly higher levels of MPV when compared with benign goiters and healthy subjects, and significant decrement was observed with tumor removal (6). However, we could not find a significant difference in MPV levels among the groups in our study.

PDW is a standard deviation of log-transformed data of platelets, and higher levels indicate that abnormally small and large platelets are in circulation. PDW is useful in showing the viability of platelets for the use of transfusion. When compared with MPV, PDW is a more reliable marker in estimating that thrombocytopenia has hypoproductive or hyperdestructive etiology (19). Authors' statements about PDW are controversial in different cancer studies. PDW was found to be significantly increased in ovarian cancer patients when compared with healthy subjects. There were studies, however, that showed significant decrement in non-small cell lung cancer, breast cancer and malignant adnexal cancer patients (12-15). In our study, we observed significantly lower values in PDW in PTC patients $(\mathrm{p}<0.01)$.

PCT is an index of platelet mass and is calculated by using PLT and MPV (20). PCT can be used to determine the need for platelet transfusion (21). Studies were limited in expressing how PCT changes in cancer patients. PCT measurements were found to be higher but not significant in epithelial ovarian cancer patients when compared with benign ovarian masses and healthy subjects (10). In our study, PCT was significantly higher in PTC patients ( $p=0.03$ ).

There are some limitations of this study. This is a retrospective study and consists of a small number of cases. Because of that, further prospective studies with large series are needed to investigate the changes of platelet indices in benign and malignant thyroid diseases.

\section{Conclusion}

This is the first study about the usage of platelet indices in the differential diagnosis of benign and malignant thyroid diseases. Lower PDW and higher PCT values were observed in papillary thyroid carcinoma patients when compared with healthy and benign disordered patients. Platelet indices may be used as useful clinical markers in the differential diagnosis of benign and malignant thyroid disorders.

\section{References}

1. Jiang H, Tian Y, Yan W et al. The Prevalence of Thyroid Nodules and an Analysis of Related Lifestyle Factors in Beijing Communities. Int J Environ Res Public Health 2016; 13 (4): 442.

2. Tuttle RM, Ball DW, Byrd D et al. Thyroid Carcinoma. J Natl Compr Canc Netw 2010; 8 (11): 1228-1274.

3. Pinchera A, Aghini-Lombardi F, Antonangeli L et al. Multi nodular goiter: epidemiology and prevention. Ann Ital Chir 1996; 67 (3): 317-325.

4. Report of the Thyroid Cancer Guidelines Update Group. Guidelines for the management of thyroid cancer British Thyroid Association, Royal College of Physicians. 2nd ed. London: 2007.

5. Başoğlu M, Karabulut K. Well-differentiated thyroid cancers. J Exp Clin Med 2012; 29 (4): 329-332.

6. Baldane S, Ipekci SH, Sozen M et al. Mean platelet volume could be a possible biomarker for papillary thyroid carcinomas. Asian Pac J Cancer Prev 2015; 16 (7): 2671-2674.

7. Mahdavi-Zafarghandi R, Shakiba B, Keramati MR et al. Platelet volume indices in patients with varicocele. Clin Exp Reprod Med 2014; 41 (2): 92-95.

8. Schwartz D, Sharkey L, Armstrong PJ et al. Platelet volume and plateletcrit in dogs with presumed primary immune-mediated thrombocytopenia. J Vet Intern Med 2014; 28 (5): 1575-1579.

9. Akpinar I, Sayin MR, Gursoy YC et al. Plateletcrit and red cell distribution width are independent predictors of the slow coronary flow phenomenon. J Cardiol 2014; 63 (2): 112-118.

10. Oncel M, Kiyici A, Oncel $M$ et al. Evaluation of platelet indices in lung cancer patients. Asian Pac J Cancer Prev 2015; 16 (17): 7599-7602.

11. Lancé MD, van Oerle R, Henskens YM et al. Do we need time adjusted mean platelet volume measurements? Lab Hematol 2010; 16 (3): 28-31.

12. Inagaki N, Kibata $\mathbf{K}$, Tamaki $\mathbf{T}$ et al. Prognostic impact of the mean platelet volume/platelet count ratio in terms of survival in advanced non-small cell lung cancer. LungCancer 2014; 83 (1): 97-101.

13. Ma X, Wang Y, Sheng $\mathbf{H}$ et al. Prognostic significance of thrombocytosis, platelet parameters and aggregation rates in epithelial ovarian cancer. J Obstet Gynaecol Res 2014; 40 (1): 178-183.

14. Ozaksit G, Tokmak A, Kalkan H et al. Value of the platelet to lymphocyte ratio in the diagnosis of ovarian neoplasms in adolescents. Asian Pac J Cancer Prev 2015; 16 (5): 2037-2041.

15. Okuturlar Y, Gunaldi M, Tiken EE et al. Utility of peripheral blood parameters in predicting breast cancer risk. Asian Pac J Cancer Prev 2015; 16 (6): 2409-2412.

16. Li JY, Li Y, Jiang Z et al. Elevated mean platelet volume is associated with presence of colon cancer. Asian Pac J Cancer Prev 2014; 15 (23): 10501-10504.

17. Coupland LA, Parish CR. Platelets, selectins, and the control of tumor metastasis. Semin Oncol 2014; 41 (3): 422-434.

18. Tuncel T, Ozgun A, Emirzeoglu L et al. Mean platelet volume as a prognostic marker in metastatic colorectal cancer patients treated with bevacizumabcombined chemotherapy. Asian Pac J Cancer Prev 2014; 15 (15): 6421-6423.

19. Alsweedan SA, Al-Shurman A, Mahmoud AS. Diagnostic value of platelet indices in children with leukemia. J Pediatr Hematol Oncol 2008; 30 (12): 953-955.

20. Ergelen M, Uyarel H. Plateletcrit: a novel prognostic marker for acute coronary syndrome. Int J Cardiol 2014; 177 (1): 161.

21. Chandrashekar V. Plateletcrit as a screening tool for detection of platelet quantitative disorders. J Hematol 2013; 2 (1): 22-26.

Received November 27, 2016. Accepted December 13, 2016. 